$$
\begin{aligned}
& \text { برمجة الارواء المثلى }
\end{aligned}
$$

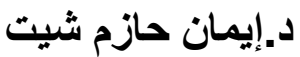

$$
\begin{aligned}
& \text { قسم الموارد المائية، كلية الهندسة، جامعة الموصل }
\end{aligned}
$$

الخلاصة الاصنة

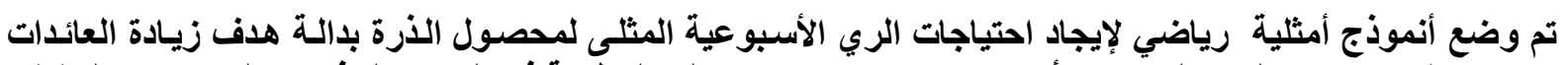

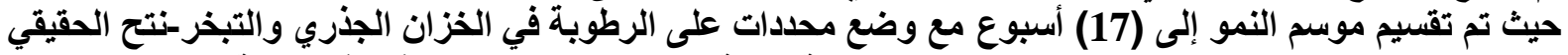

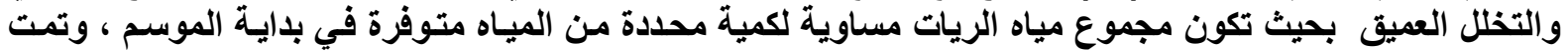

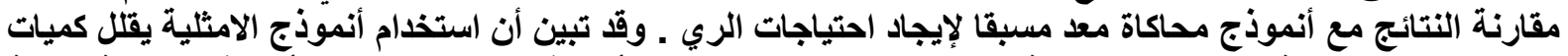

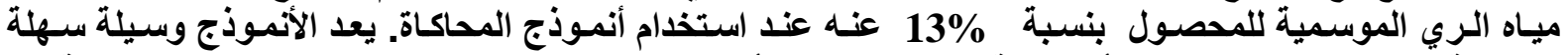

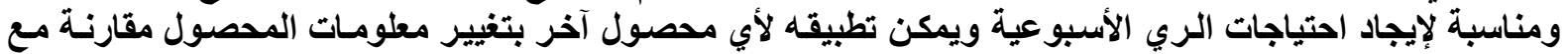

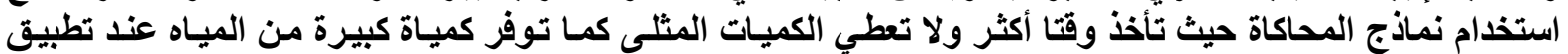
الأنموذج لعدة محاصيل ولمساحات كبيرة.

الكلمات الدالة: الامثلية، جدولة الارواء، الإنتاجية المثلى

\title{
OPTIMAL IRRIGATION PROGRAMMING
}

\author{
Dr.Eman Hazim Sheet \\ Water Resource Dept., College of Eng., Mosul Univ.
}

\begin{abstract}
A mathematical optimization model for optimum weekly irrigation water requirements for maize crop at Mosul area is developed. The main objective function is to maximize profit. The growing season is divided into (17)weeks with constraints on actual evapotranspiration, soil moisture at the root zone and deep percolation. The total amount of irrigation water requirements is assumed known and limited at the beginning of the season. The results of this approach was compared with a previous simulation model for irrigation water requirement. It is found that optimization model reduce seasonal irrigation water by $13 \%$ compared with simulation models. The proposed model is simple for predicting weekly irrigation requirements and can be used for any crop by changing crop data. where simulation models take longer time and do not give optimal solutions. As this model is applied to many crops grown over large areas, a huge amount of irrigation water may be saved
\end{abstract}

KEYWORDS:optimization, irrigation scheduling, optimal yield 


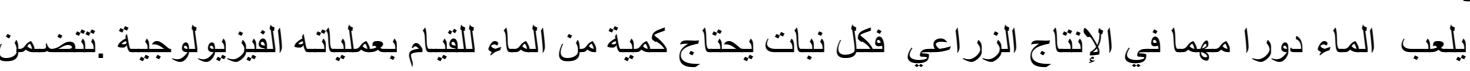

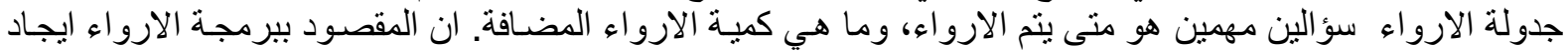

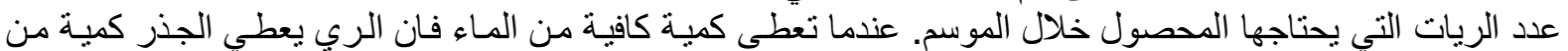

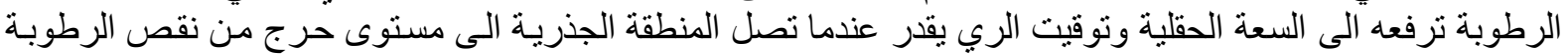

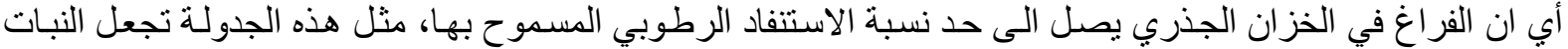

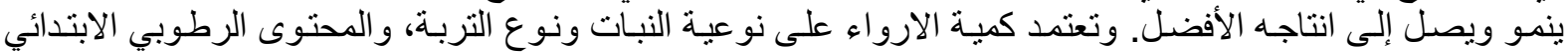

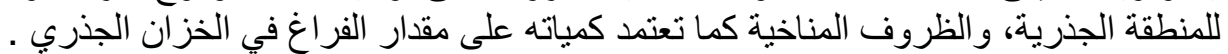

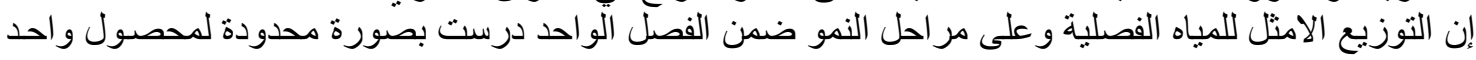

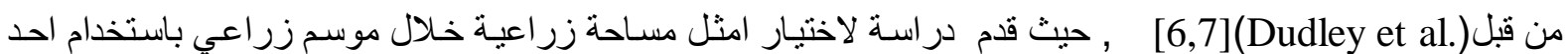
نماذج البرمجة الدينامية ( Dynamic programming ) وربط ذللك بتوفير خزين مائي في بحيرة, واستنتج أن أفضل

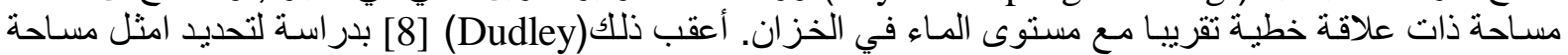

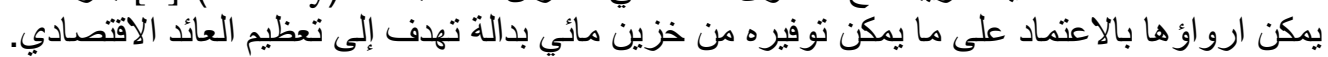

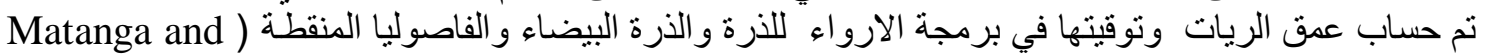
(Marino

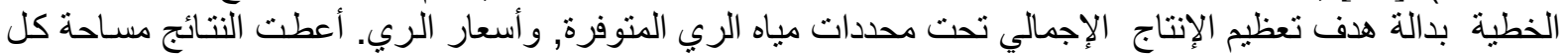

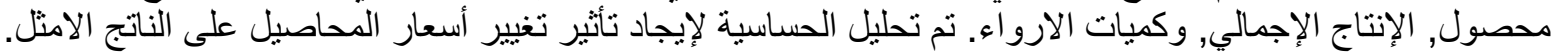

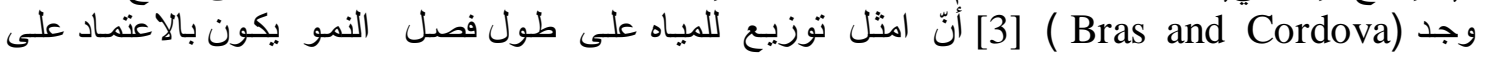

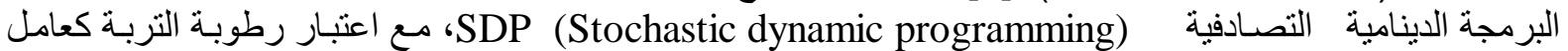
عشو ائي. أما (10) (10) فقد قاموا بتحليل معلومات عن حقل مزروع بثلاثة أنواع من المحاصيل، منها تعتمد

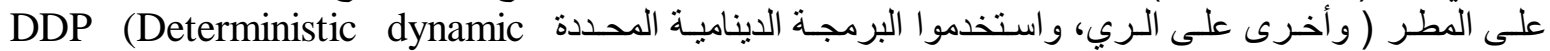
programming)

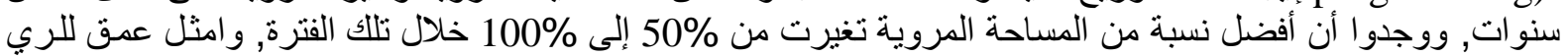

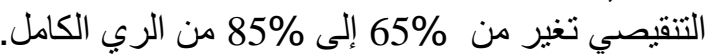

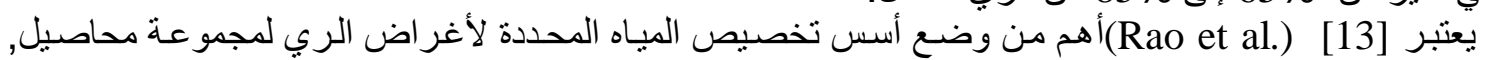

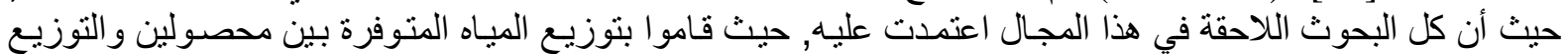

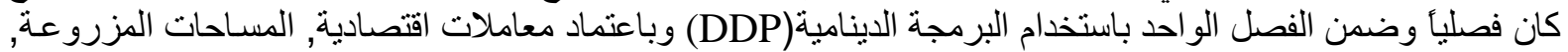

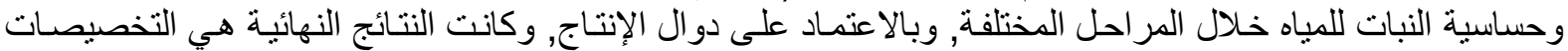

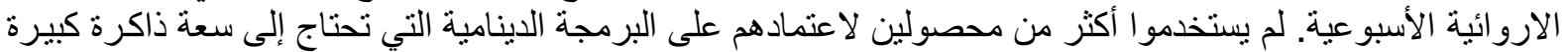

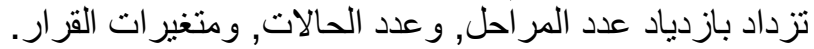

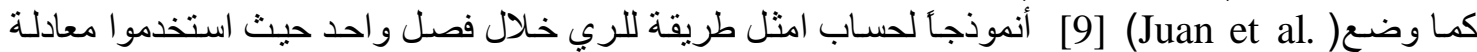

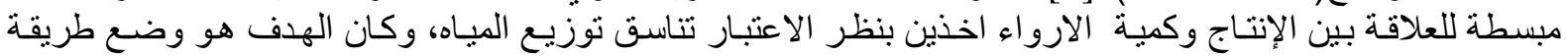
مبسطة يمكن استخدامها من قبل المز النارع.

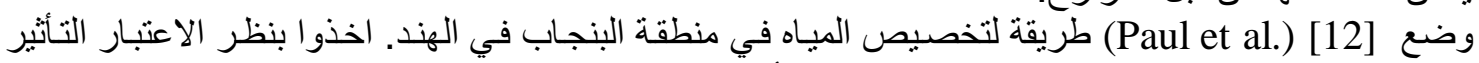

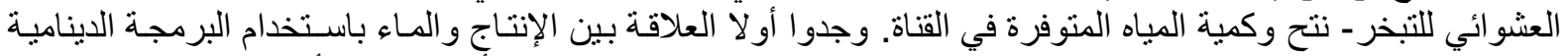

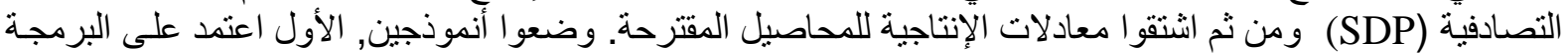

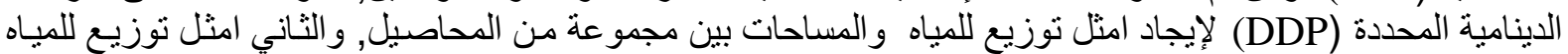

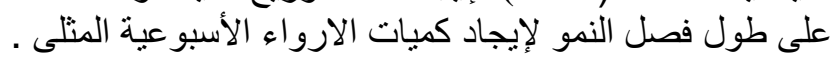

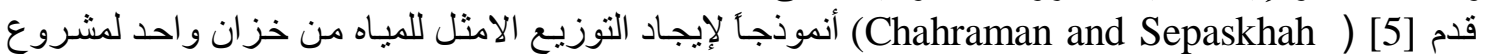

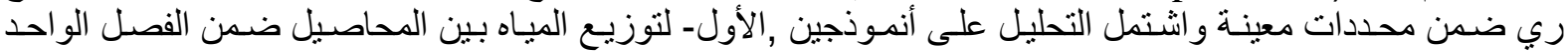

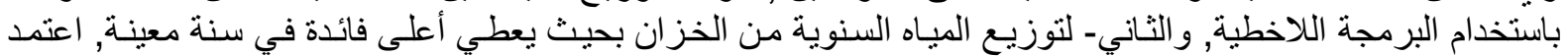

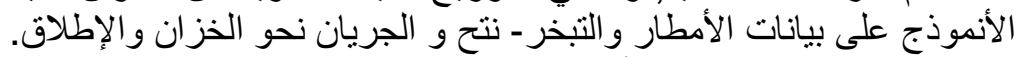

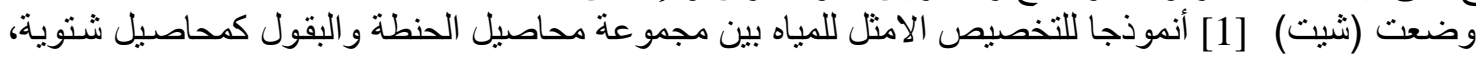

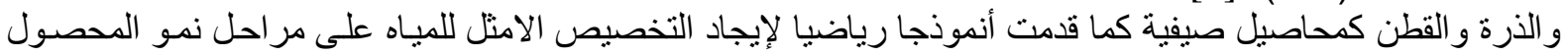

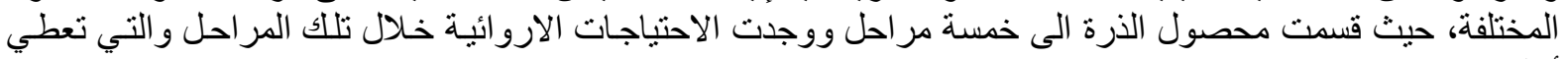

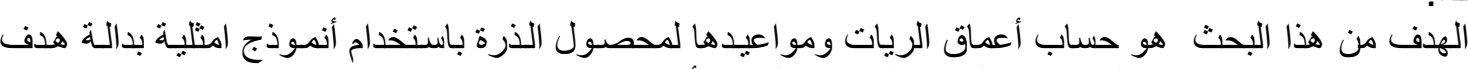
أعلى فائدة. زيادة العائدات ، كما تمت مقارنة النتائج مع النتائج المستحصلة من أنمات أنموذج محاكاة. 
طرق ومواد البحث

تم وضع أنموذج امثلية بدالة هدف تعظيم العائدات لإيجاد التخصيصات الاروائية الأسبو عية لمحصول الذرة خلال

$M A X . . N B=A .\left[B^{*} Y-C\right]$

الأنموذج (1) بدالة هدف تعظيم العائدات:

حيث أنّ:

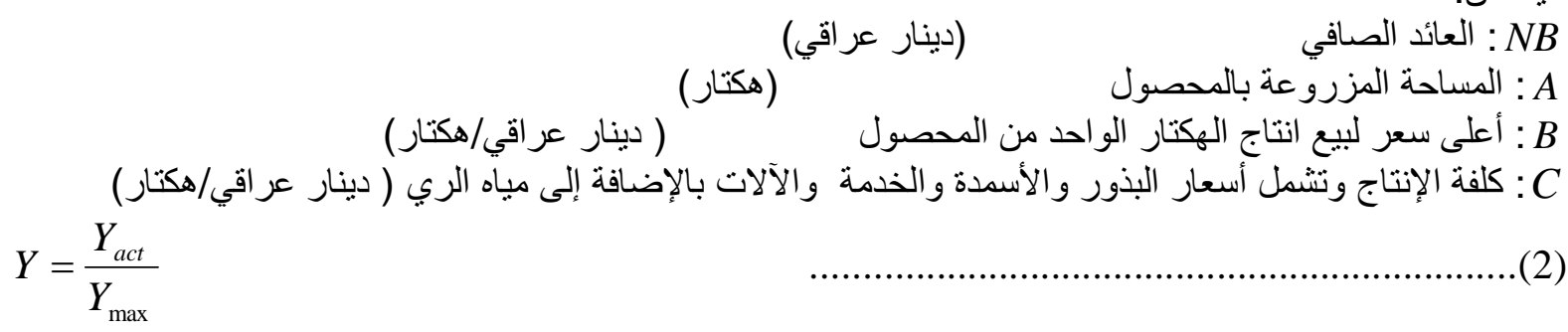

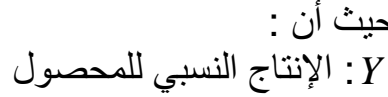
( بct

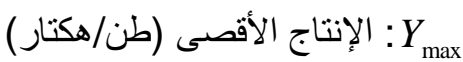

$$
T N B=A .\left[B *\left\{\prod_{j=1}^{n}\left(1-k y_{j}\left(1-\frac{A E T_{j}}{C P E T_{j}}\right)\right)\right\}-C\right]
$$

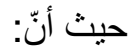

(TNB

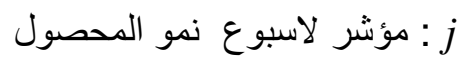

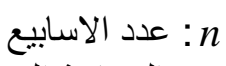

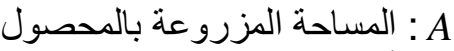

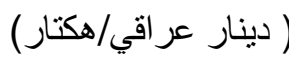

(ملم)

( ) - (دينار عر اقي/هكتار )

$C=a+b(X)$

(وتساوي 76 ألف دينار /هكتار)

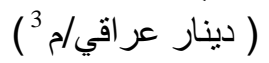

B : أعلى سعر لبيع انتاج الهكتار الو احد من المحصول

(وتساوي 450 ألف دينار /هكتار)

ا

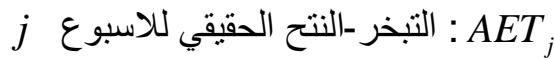

j

(C) - كلفة الإنتاج للمحصول

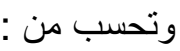

حيث أنّ : a : ثابت بمثل أسعار الذذور و الأسمدة والخدمة والآلات

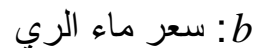
X

$S M_{j+1} R Z_{j+1}=S M_{j} R Z_{j}+X_{j}+\operatorname{Rain}_{j}-A E T_{j}+S M_{\circ}\left(R Z_{j+1}-R Z_{j}\right)-D P_{j} .$.

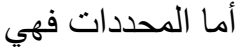

$j=1,2,3,4,5$,

$A E T_{j}<=C P E T$ 


$$
\begin{aligned}
& A E T_{j}<=\frac{S M_{j}}{(1-p) T A W_{j}}{C P E T_{j}} \\
& S M_{j}<=F C \\
& S M_{j}>=P W P . \\
& D P_{j}<=(1-A E) X_{j}
\end{aligned}
$$

$$
\begin{aligned}
& \text { حيث أنّ: }
\end{aligned}
$$

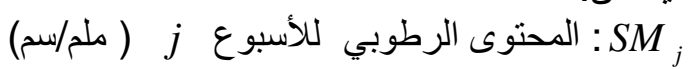

$$
\begin{aligned}
& \text { : المحتوى الرطوبي الابتدائي ( ملم/سم) : : : } \\
& \text { : : } X_{j}
\end{aligned}
$$

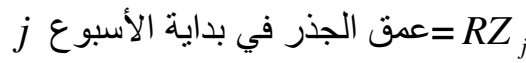

p = نسبة من الماء الكلي المنيسر الذي يمكن أن يستنفذ من الجذر قبل التعرض للإجهاد (=0.55 لمحصول الذرة) [2] .

( مل: DP

(\% : كفاءة إضافة المياه ( A

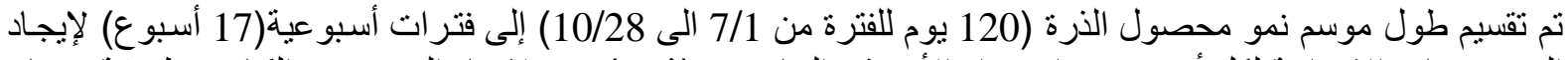

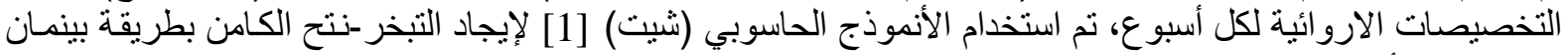

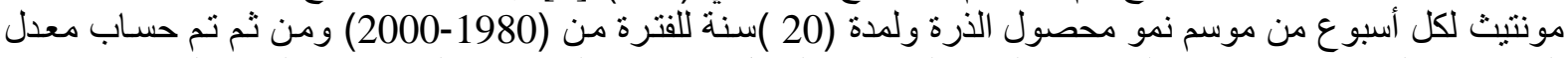

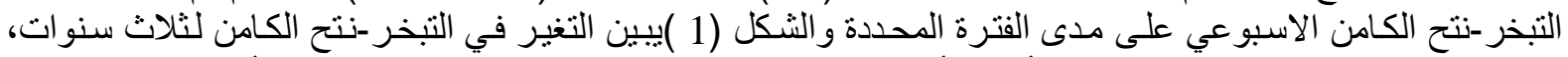

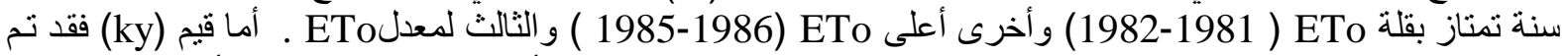
إيجادها لمر احل نمو محصول الذرة (Allen) [2] وتم توزيعها على الفترات الأسبو عية لعدم توفر قيم أسبو عية لمعامل

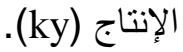

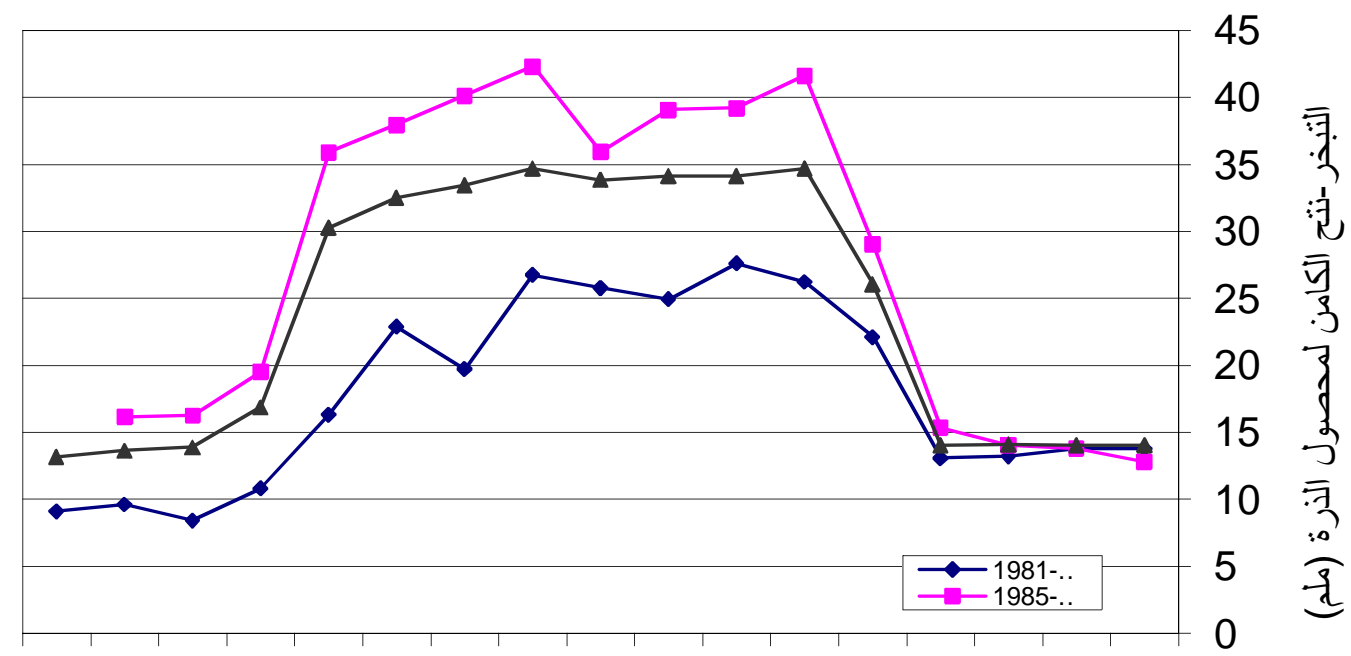

$\begin{array}{lllllllllllllllll}17 & 16 & 15 & 14 & 13 & 12 & 11 & 10 & 9 & 8 & 7 & 6 & 5 & 4 & 3 & 2 & 1\end{array}$

الثكل(1): التبخر -نتح الكامن الاسبو عي لمحصول الذرة في منطقة الموصل لثنلاث سنو ات جافة ورطبة ولمعدل التبخر نتح الكامن لمدة(20)سنة

(Borg and Grimes) ${ }^{[4]}$ (إيجاد تغير عمق الجذر لكل أسبوع من معادلة

$$
R Z(I)=M R D\left[0.5+0.5 \sin \left\{3.03\left(\frac{I}{D m}\right)-1.47\right\}\right]
$$




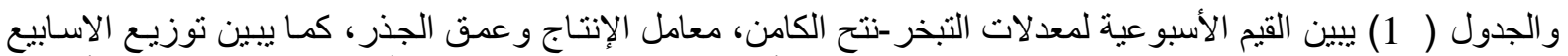

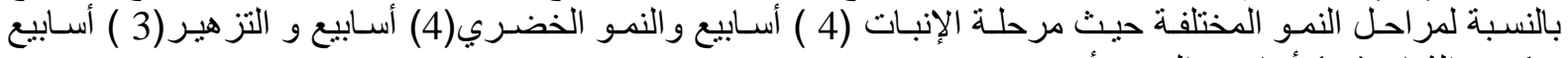
وتكوين الثمار (5 ) أسابيع و النضهج أسبوع.

الجدول(1): القيم الأسبوعية لمعدلات التبخر -نتح الكامن لفترة(20) سنة ، معامل الإنتاج، معامل محصول الذرة وعمق الجذر الترن

\begin{tabular}{|c|c|c|c|c|c|}
\hline الجذر & $\begin{array}{l}\text { معامل الإنتاج } \\
\text { ky }\end{array}$ & 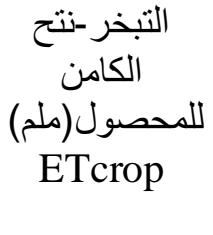 & محصول & مر احل & الاسبوع \\
\hline 7 & \multirow{8}{*}{0.4} & 14 & \multirow[t]{4}{*}{0.4} & \multirow{4}{*}{ (الانباتى) } & 1 \\
\hline 20 & & 14 & & & 2 \\
\hline 40 & & 14 & & & 3 \\
\hline 62 & & 14 & & & 4 \\
\hline 85 & & 26 & \multirow[t]{4}{*}{1.0} & \multirow{4}{*}{$\begin{array}{c}\text { الخضرية (النمو } \\
\text { الخانية) }\end{array}$} & 5 \\
\hline 107 & & 35 & & & 6 \\
\hline 121 & & 34 & & & 7 \\
\hline \multirow{10}{*}{135} & & 34 & & & 8 \\
\hline & \multirow{3}{*}{1.5} & 34 & \multirow[t]{4}{*}{1.1} & \multirow{3}{*}{ (الثز هير ) } & 9 \\
\hline & & 35 & & & 10 \\
\hline & & 33 & & & 11 \\
\hline & \multirow{6}{*}{0.5} & 32 & & \multirow{5}{*}{$\begin{array}{l}\text { التكوابعة } \\
\text { الثمار) }\end{array}$} & 12 \\
\hline & & 30 & \multirow[t]{5}{*}{0.55} & & 13 \\
\hline & & 17 & & & 14 \\
\hline & & 14 & & & 15 \\
\hline & & 14 & & & 16 \\
\hline & & 13 & & (النضامسة & 17 \\
\hline
\end{tabular}

تم اعتبار المحتوى الرطوبي الابتدائي عند السعة الحقلية لتربة متوسطة النسجة (3.1 ملم/سم) ونقطة الذبول الدائم (1.5

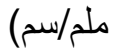
محددات الموازنة المائية للخزان الجذري

$$
S M_{1} R Z_{1}=3.1 * 7=21.7
$$

$$
S M_{2} R Z_{2}=3.1 * 7.0+X_{1}+3.1(20-7)-A E T_{1}-D P_{1}
$$


$S M_{2} * 20=62+X_{1}-A E T_{1}-D P_{1}$

$S M_{2}=\left(62+X_{1}-A E T_{1}-D P_{1}\right) / 20$

$\mathrm{SM} 3=(20 * \mathrm{SM} 2+\mathrm{X} 2-\mathrm{AET} 2+62-\mathrm{D} 2) / 40$;

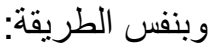

$\mathrm{SM} 4=(40 * \mathrm{SM} 3+\mathrm{X} 3-\mathrm{AET} 3+68.2-\mathrm{D} 3) / 62$

$\mathrm{SM} 5=(62 * \mathrm{SM} 4+\mathrm{X} 4-\mathrm{AET} 4+71.3-\mathrm{D} 4) / 85$;

SM6 $=(85 *$ SM5+X5-AET5+68.3-D5)/107;

SM7 $=(107 *$ SM6+X6-AET6+43.4-D6)/121;

$\mathrm{SM} 8=(121 * \mathrm{SM} 7+\mathrm{X} 7-\mathrm{AET} 7+43.4-\mathrm{D} 7) / 135$;

$\mathrm{SM} 9=(135 * \mathrm{SM} 8+\mathrm{X} 8-\mathrm{AET} 8-\mathrm{D} 8) / 135$

$\mathrm{SM} 10=(135 * \mathrm{SM} 9+\mathrm{X} 9-\mathrm{AET} 9-\mathrm{D} 9) / 135$;

$\mathrm{SMi}=\left(135 * \mathrm{SM}_{\mathrm{i}-1}+\mathrm{X}_{\mathrm{i}-1}-\mathrm{AET}_{\mathrm{i}-1}-\mathrm{D}_{\mathrm{i}-1} / 135\right.$; for $i=11-17$

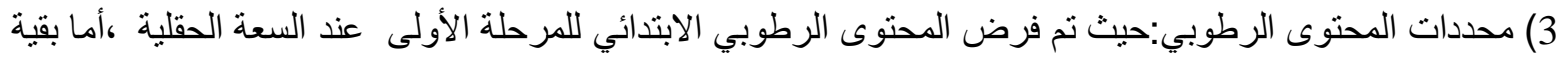

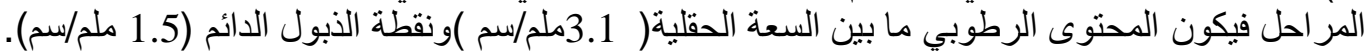

$S M_{1}=3.1$

$3.1>=S M_{i}>=1.5$

$i=2,3,4$.

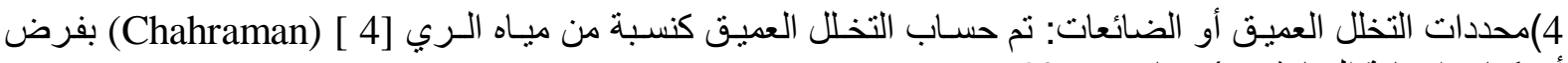

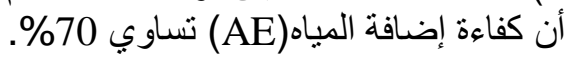

$D P_{i}<=(1-0.7) X_{i}$

$D P_{i}<=0.3 * X_{i}$

$i=1,2,3$

الأنموذج الثاني، أنموذج المحاكاة: تم تطبيق أنموذج المحاكاة لبرمجـة الإرواء اليوميـة (شيت) [1] لإيجاد الاحتياجات

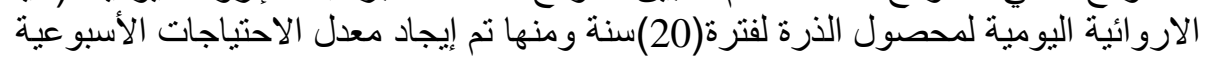

النتائج و المناقثنة

تم تنفيذ الأنموذج بدالة هدف زيادة العائدات، باستخدام برنامج (LINGO )وكانت الحئ نتائج الأنموذج هي الريات الأسبو عية

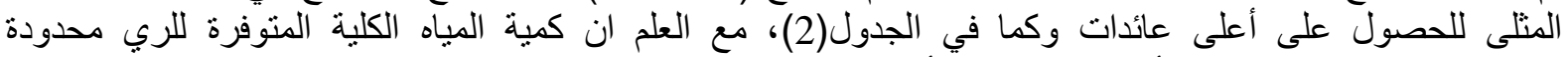

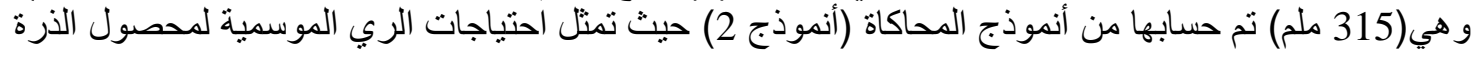


الجدول(2): الريات الأسبو عية والمقترحة المتلى للأنموذجين الاول و الثاني

\begin{tabular}{|c|c|c|c|c|}
\hline \multicolumn{2}{|c|}{ أنموذج (2) } & \multicolumn{2}{|c|}{ أنموذج (1) } & \multirow[t]{2}{*}{ الاسابيع } \\
\hline الريات المقترحة & الريات الأسبو عية & الريات المقترحة & الريات الأسبو عية & \\
\hline \multirow{3}{*}{40} & 5 & \multirow{3}{*}{40} & 3.0 & 1 \\
\hline & 30 & & 3.0 & 2 \\
\hline & 7 & & 36.0 & 3 \\
\hline \multirow{3}{*}{79} & 7 & \multirow{3}{*}{60} & 5.0 & 4 \\
\hline & 32 & & 35.0 & 5 \\
\hline & 40 & & 16.5 & 6 \\
\hline \multirow[b]{2}{*}{101} & 43 & 78 & 78.0 & 7 \\
\hline & 58 & \multirow[b]{2}{*}{63} & 10.0 & 8 \\
\hline \multirow{10}{*}{93} & 16 & & 53.0 & 9 \\
\hline & 55 & \multirow{8}{*}{76} & 28.0 & 10 \\
\hline & 18 & & 48.0 & 11 \\
\hline & 3.5 & & 0.00 & 12 \\
\hline & 0 & & 0.00 & 13 \\
\hline & 0 & & 0 & 14 \\
\hline & 0 & & 0 & 15 \\
\hline & 0 & & 0 & 16 \\
\hline & 0 & & 0 & 17 \\
\hline & 315.5 & & 315.5 & المجموع \\
\hline
\end{tabular}

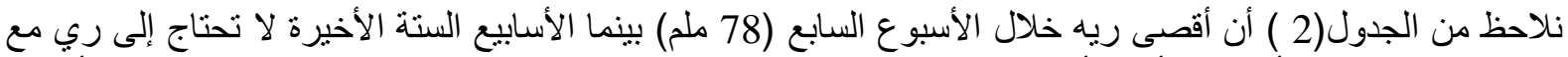

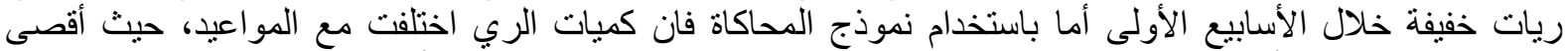

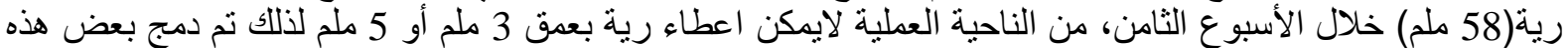

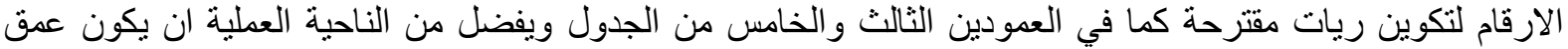

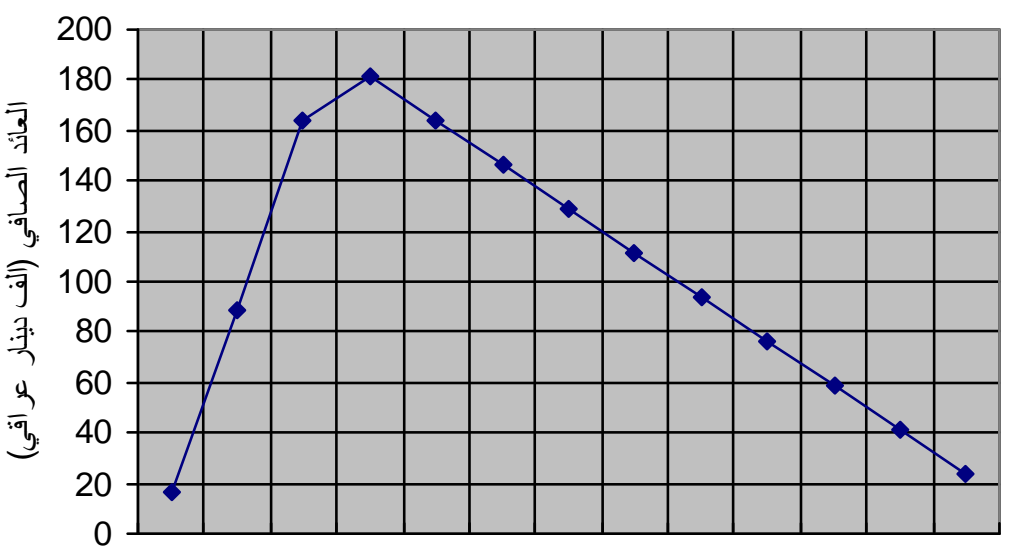

200225250275300325350375400425450475500

عمق الارواء الموسمى (ملم)

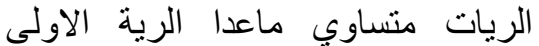

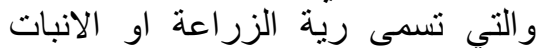

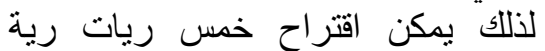

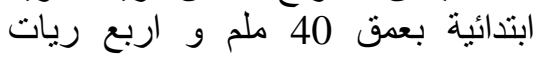

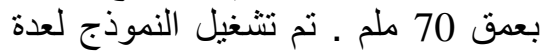

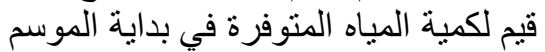

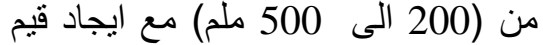
العائدات الصافية وتبين أن الحل الأمثل

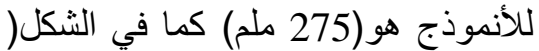

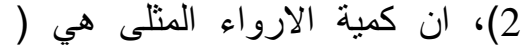

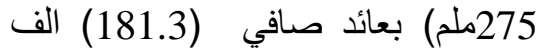
دينار عراقي، ، بينما عند زيادة كمية الفية المياه تقل العائدات نظرا لزيادة لنياد تكاليف

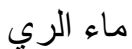
الشكل(2):تغير العائد الصافي بتغيير عمق الارواء الرواء الموسمي 


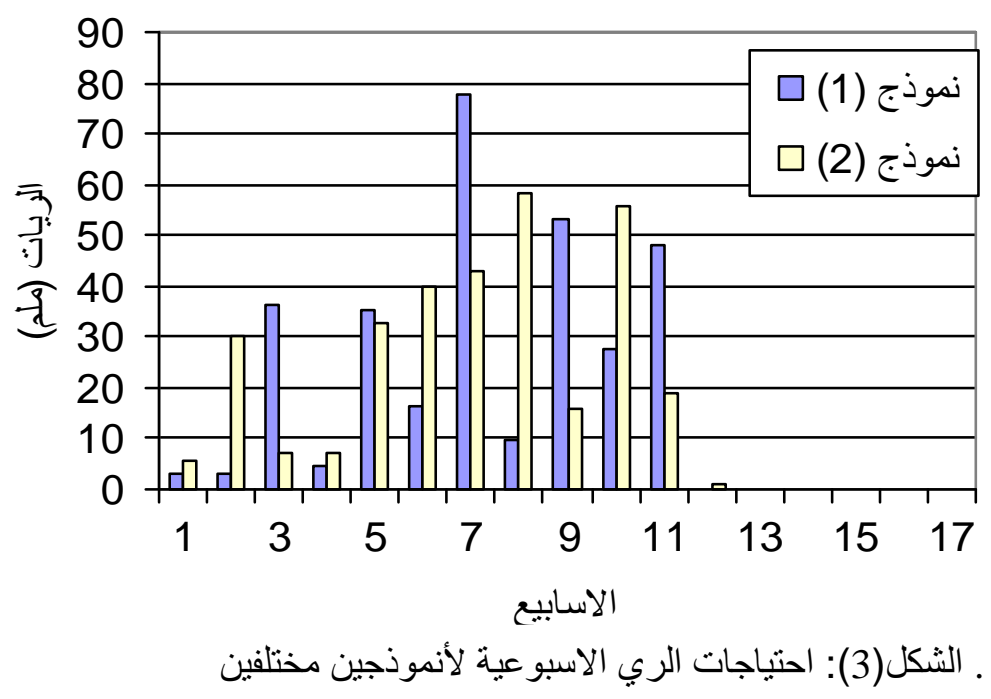

يمكن اعتبار الأنموذج طريقة سهلة ومبسطة لحساب كميات الارواء لاء لاء

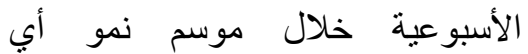
محصول باستخدام الطريقة المبينة كماً تمت مقارنة أنموذج الامثلية مع أنموذج الطنجة

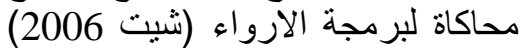

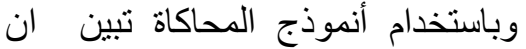
احتياجات الري الموسمية لمحصول النيات

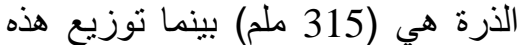
الريات سيتغير خلال الموسم كما في الثكل ( 3) لنموذجين مختلفين.

1. ششيت، إيمان حازم (2006).” التخطيط الأمثل للري ألتنقيصي في منطقة الموصل “، اطروحة دكتور اه ،كلية الهندسة، قسم الموارد المائية، جامعة الموصل.

2. Allen, R. G. ,L.S. Pereira, D. Raes and M.Smith (1998). “ Crop evapotranspiration-Guidelines for computing crop water requirements ".FAO Irrigation and Drainage paper No.56, Rome, Italy.

3. Bras, L. R. and J. R. Cordova (1981). "Intraseasonal water allocation in deficit irrigation". Water Resources Research, 17(4): 866-874.

4. Borg, H. and D.W.Grimes (1986). "Depth development of roots with time: An empirical description ".Trans. ASAE. 29(1), 194-197.

5. Chahraman, B. and A. Sepaskhah (2002). "Optimal allocation of water from a single reservoir to an irrigation project with pre-determined multiple cropping pattern". Irrigation Science, 21: 127-137.

6. Dudley, N. J., D. T. Howell and W. D. Musgrave(1971,a). "Irrigation planning 2. Choosing optimal acreages within a season". Water Resources Research , 7(5): 1051-1063.

7. Dudley, N. J., D. T. Howell and W. D. Musgrave(1971,b). "Irrigation planning 3. The best size of irrigation area for a reservoir". Water Resources Research , 8(1): 7-17

8. Dudley, N. J. (1972). "Irrigation planning 4:Optimal intraseasonal water allocation". Water Resources Research, 8(3)586-594.

9. Juan, J. A., J. M. Tarjuelo, P. Garcia, and M. Valiente (1996). "A model for optimal cropping patterns within the farm based on crop water production functions and irrigation uniformity. I: Development of decision model". Agriculture Water Management, 31: 115-143.

10. Martin, D. L ., J. Brocklin and G. Wilmes $(1989, a)$. "Operating rules for deficit irrigation". Trans. ASAE 32(4): 1207-1215.

11. Matanga, G. B. and M. A. Marino (1979). "Irrigation planning 1. Cropping pattern" . Water Resources Research , 15(3): 627-678.

12. Paul, S., S. M. Panda and D. N. Kumar (2000). "Optimal irrigation allocation, a multilevel approach". Journal of the Irrigation and Drainage Division ASCE. 126(3): 149-156.

13. Rao, N. H. , P. B. S. Sarma and S. Chander (1990). "Optimal multicrop allocation ofseasonal and intraseasonal irrigation water". Water Resources Research , 26(4): 551-559.

$$
\text { تم اجراء البحث في كلية الهندسة - جامعة الموصل }
$$

